\title{
Power and Sample Size Calculations with the Additive Hazards Model
}

\author{
Ling Chen*, Chengjie Xiong, J. Philip Miller and Feng Gao \\ Washington University School of Medicine
}

\begin{abstract}
Existing methods on sample size calculations for right-censored data largely assume the failure times follow exponential distribution or the Cox proportional hazards model. Methods under the additive hazards model are scarce. Motivated by a well known example of right-censored failure time data which the additive hazards model fits better than the Cox model, we proposed a method for power and sample size calculation for a two-group comparison assuming the additive hazards model. This model allows the investigator to specify a group difference in terms of a hazard difference and choose increasing, constant or decreasing baseline hazards. The power computation is based on the Wald test. Extensive simulation studies are performed to demonstrate the performance of the proposed approach. Our simulation also shows substantially decreased power if the additive hazards models is misspecified as the Cox proportional hazards model.
\end{abstract}

Key words: Additive hazards model, power, right-censored data, sample size.

\section{Introduction}

The Cox proportional hazards (PH) model (Cox, 1972) has become the standard tool and routinely used in the analysis of survival data. The hazard function for the failure time $T$ associated with a $p$-vector of possibly time-varying covarites $Z(t)$ takes the form

$$
\lambda(t ; Z)=\lambda_{0}(t) e^{\gamma^{\prime} Z(t)},
$$

where $\gamma$ denotes a $p$-vector of unknown regression parameters and $\lambda_{0}(t)$ is an unspecified baseline hazard function. A useful alternative to the Cox model is the Aalen additive hazards model (Aalen, 1980) which takes the form

$$
\lambda(t ; Z)=\lambda_{0}(t)+\beta^{\prime} Z(t),
$$

\footnotetext{
${ }^{*}$ Corresponding author.
} 
where $\beta$ denotes a $p$-vector of unknown regression parameters. These models provide two principal frameworks for studying the association between risk factors and disease occurrence or death, namely, the multiplicative effect of risk factors on the hazard of the failure time for the Cox model and the additive effect for the Aalen model.

The partial likelihood approach introduced by Cox $(1972,1975)$ eliminates the nuisance quantity $\lambda_{0}(t)$ from the score function for $\gamma$. The resulting maximum partial likelihood estimator possesses asymptotic properties similar to those of the standard maximum likelihood estimator (Tsiatis, 1981; Anderson and Gill, 1982). Such desirable theoretical properties, together with the wide availability of computer programs, have made the Cox model a popular choice in many fields of survival analysis. However, little emphasis has been placed on the fit of the model. Additionally, under the PH model the effect of covariates is restricted to be multiplicative on hazards of failure. The additive hazards model was argued to be more reasonable for certain instances such as dose on risk or hazard (Breslow and Day, 1987) and the covariate effect directly reflects the difference of hazards and is thus easier to interpret. It is important to decide which of the two models that are more appropriate to apply in a given application. This is nontrivial as these two classes of models are not nested except for special cases.

Sample size calculations have been an important part in the design of clinical trials. The sample size calculations in survival analysis of right-censored failure times are largely based on exponential distribution or the Cox model (Schoenfeld, 1983). However, there exists no literature on sample size or power calculation assuming the additive hazards model. It is well known that a sample size calculation under a misspecified model may yield compromised power or increased sample size.

Several methods have been proposed to compare the fit of the two models. Among these, one method is to embed the two models within a power family asymmetric transformations of the linear predictor proposed by Aranda-Ordaz (1981, 1983) for grouped survival data. This family transformation has as special cases the Cox and additive hazards models. In his paper, a score test statistic was developed to discriminate between these models which is asymptotically equivalent to maximum likelihood ratio test. Tibshirani and Ciampi (1983) generalized Aranda-Ordaz's family transformation to allow time trends in the hazards. McKeague and Utikal (1991) introduced a goodness-of-fit test for the Cox and additive hazards models. They proposed goodness-of-fit statistics based on differences between the estimates of a doubly cumulative hazard function and a fully nonparametric estimators of this cumulative hazard function under both models. Recently, Martinussen, Aalen and Scheike (2008) proposed a Mizon-Richard emcompassing test for this problem which corresponds to fitting the additive hazards 
model to the martingale residues obtained from the Cox regression analysis.

As the motivation of this paper, we present a well-known example of rightcensored failure time data which can be fit better by the additive hazards model than the Cox model. These data arose from a clinical trial of drug 6-Mercaptopurine (6-MP) versus a placebo in 42 children with acute leukemia reported by Freireich et al. (1963) and used by Gehan (1965) and several subsequent authors. Table 1 shows the ordered length of remission for two samples of individuals with censored values indicated by plus.

Table 1: Lengths of Remission (in weeks) for leukemia patients (from Gehan, 1965)

\begin{tabular}{llllllllllll}
\hline 6 -MP & 6 & 6 & 6 & $6^{+}$ & 7 & $9^{+}$ & 10 & $10^{+}$ & $11^{+}$ & 13 & 16 \\
& $17^{+}$ & $19^{+}$ & $20^{+}$ & 22 & 23 & $25^{+}$ & $32^{+}$ & $32^{+}$ & $34^{+}$ & $35^{+}$ & \\
\hline Placebo & 1 & 1 & 2 & 2 & 3 & 4 & 4 & 5 & 5 & 8 & 8 \\
& 8 & 8 & 11 & 11 & 12 & 12 & 15 & 17 & 22 & 23 & \\
\hline
\end{tabular}

Because of the computational simplicity, we applied Aranda-Ordaz's efficient score test to this data set. This method uses a multiplicative grouping model and can accommodate ties of event times. Five partition intervals (0-5, 5-10, 10-15, 15-20 and 20-25) are used to group the time of remission for leukemia patients. At a significance level of 0.05, a two-sided test for departure from the Cox model yields a Z-score 7.24 ( $p$-value $<0.0001)$. However, a two-sided test for departure from the additive hazards model yields a Z-score $-1.13(p$-value $=0.258)$. These tests provide strong evidence that the Additive hazards model is a better fit than the Cox model in describing the effect of 6 -MP on the hazard of remission time in patients with acute leukemia. Based on these pilot data, if clinicians would like to design a pivotal study to compare 6-MP to placebo where the outcome is time to remission in patients with acute leukemia, a method for sample size or power calculation under the additive hazards model is warranted.

We propose a method for power calculations for clinical trials comparing two groups with right-censored data under the additive hazards model in Section 2. We consider the additive hazards model with the baseline hazard $\lambda_{0}(t)$ from a Weilbull random variable. This specification allows constant, decreasing and increasing baseline hazards which describes many disease processes and is appealing to medical investigators. In Section 3, we illustrate the proposed method using the leukemia example described above. Section 4 presents simulation studies to demonstrate the performance of the proposed approach, followed by discussion and concluding remarks in Section 5 .

\section{Methods}


Consider a survival study and let $T$ denote the failure time of interest and $Z$ a vector of covariates that may depend on time $t$. We assume that right-censored failure time data are available and given by $\left\{X_{i}, \delta_{i}, Z_{i}, i=1, \cdots, N\right\}$ from $N$ independent subjects. Here $X_{i}$ denotes the observed failure time defined as the minimum of the true failure time $T_{i}$ and the censoring time $C_{i}$ for subject $i$ and $\delta_{i}=1$ if the true failure time is observed and 0 censored. It is assumed that the failure time and the censoring time are independent given covariates.

Assume the failure time $T$ follows the additive hazards model with the baseline hazard following a Weibull distribution with a scale parameter $\theta$ and a shape parameter $\nu$ with $\theta, \nu>0$. In this paper we consider a two-group comparison scenario where the covariate $Z$ is time-invariant. Given the covariate $Z$, the additive hazards model takes the form

$$
\lambda(t ; Z)=\frac{\nu}{\theta^{\nu}} t^{\nu-1}+\beta^{\prime} Z .
$$

With $0<\nu<1$ the baseline hazard is decreasing from $\infty$ to 0 , and with $\nu>1$ the baseline hazard is increasing from 0 to $\infty$. The covariate effect is to additively increase or decrease the hazard of the failure.

Let the survival distribution for the failure time $T$ be denoted by $S(t ; \theta, \nu, \beta)=$ $\operatorname{Pr}(T \geq t)$, where $t \geq 0$. The likelihood for the set of right-censored failure time data is

$$
L(X, \delta, Z ; \theta, \nu, \beta)=\prod_{i=1}^{N} S_{T}\left(x_{i}, \delta_{i}, z_{i} ; \theta, \nu, \beta\right)^{1-\delta_{i}} f_{T}\left(x_{i}, \delta_{i}, z_{i} ; \theta, \nu, \beta\right)^{\delta_{i}} .
$$

The parameters can be estimated by solving the score function $\partial L / \partial \eta=0$, where $\eta=[\theta, \nu, \beta]^{\prime}$. Since the parameters $\theta$ and $\nu$ have linear inequality constraints $(\theta, \nu>0)$, a linearly constrained optimization called adaptive barrier algorithm (constrOptim in R) can be used to estimate the parameter vector $\eta$. The derivation of the score vector and Hessian matrix is presented in Appendix.

For many sample size calculations in medical and public health settings with survival outcome, it is of main interest to compare two groups (e.g., treatment versus placebo) or two exposures (e.g., exposed versus unexposed) on the failure time. Therefore, we have a binary covariate $Z$ which usually does not change with time. Here $\beta$ represents the difference in hazard between the treatment and placebo groups. Suppose we have $n$ subjects in the placebo group $(Z=0)$ and $r n$ subjects $(r>0)$ in the treatment group $(Z=1)$ with a total of $N(n+r n=N)$. The hypothesis of interest is $H_{0}: \beta=0$ versus $H_{1}: \beta \neq 0$. We propose a power and sample size calculation based on the Wald test. The statistic of Wald test is given by

$$
T_{\text {Wald }}=\frac{\hat{\beta}^{2}}{\operatorname{var}(\hat{\beta})},
$$


where $\hat{\beta}$ is the maximum likelihood estimate of $\beta$.

Under $H_{0}$, the test statistic is asymptotically distributed as a central chisquare random variable with one degree of freedom. According to Wald (1943), $T_{\text {Wald }}$ is asymptotically distributed as noncentral $\chi_{1, \omega}^{2}$ under $H_{1}$, where the noncentrality parameter $\omega$ is the test statistic $T_{\text {Wald }}=\hat{\beta}^{2} / \operatorname{var}(\hat{\beta})$. Let $\alpha$ represent the specified type I error rate and $\chi_{1,1-\alpha}^{2}$ represent the critical value from the central $\chi_{1}^{2}$ distribution. The power for testing $H_{0}$ with the Wald test is

$$
1-\phi=\int_{\chi_{1,1-\alpha}^{2}}^{\infty} \frac{e^{-\left(s^{2}+\omega^{2}\right) / 2} s \omega}{\sqrt{\omega s}} I_{-1 / 2}(\omega s) d s,
$$

where $\phi$ is the type II error rate and $I_{\nu}($.$) is a modified Bessel function of the$ first kind. (2.2) can be calculated in $\mathrm{R}$ (function pchisq).

To calculate the power for the study, we need to first specify a clinically meaningful covariate effect $\beta$ between the two groups. To estimate $\operatorname{var}(\hat{\beta})$, we need to calculate the expected value of the second derivative matrix

$$
E\left[-\sum_{i=1}^{N} \partial^{2} \log L\left(x_{i}, \delta_{i}, z_{i} ; \eta\right) / \partial \eta^{2}\right],
$$

and take the corresponding element of the matrix's inverse. To accomplish this we then specify a distribution for the censoring variable $C$. The expected value of the second derivative matrix is

$$
\begin{aligned}
E & {\left[-\sum_{i=1}^{N} \partial^{2} \log L\left(x_{i}, \delta_{i}, z_{i} ; \eta\right) / \partial \eta^{2}\right] } \\
= & -\sum_{i=1}^{N} \int_{0}^{\infty} \partial^{2}\left[\log S\left(x_{i}, \delta_{i}, z_{i} ; \eta\right)\right] / \partial \eta^{2} S_{T}\left(x_{i}\right) f_{C}\left(x_{i}\right) d x_{i} \\
& +\left\{-\sum_{i=1}^{N} \int_{0}^{\infty} \partial^{2}\left[\delta_{i} \log f\left(x_{i}, \delta_{i}, z_{i} ; \eta\right)\right] / \partial \eta^{2} f_{T}\left(x_{i}\right) S_{C}\left(x_{i}\right) d x_{i}\right\},
\end{aligned}
$$

where $S_{T}$ and $f_{T}$ are the survival and density of the failure times, $S_{C}$ and $f_{C}$ are the survival and density of the censoring times.

We can assume the censoring time $C$ follows a certain distribution. For example, if the censoring times are assumed to occur at the same hazard rate throughout the study, then an exponential distribution with parameter $\lambda_{C}$ may be appropriate. Accordingly, the probability density function for the censoring time $c_{i}$ is $f_{C}\left(c_{i}\right)=\lambda_{C} e^{-\lambda_{C} c_{i}}$. Assuming the sample size ratio of treatment $\left(z_{i}=1\right)$ to placebo $\left(z_{i}=0\right)$ is $r$, to determine a value for $\lambda_{C}$, one can specify the 
expected proportion of total failures in the study, which is

$$
\int_{0}^{\infty}\left[\frac{1}{1+r} f_{T}(t \mid z=0)+\frac{r}{1+r} f_{T}(t \mid z=1)\right] S_{C}(t) d t
$$

where $f_{T}(t \mid z=0)$ and $f_{T}(t \mid z=1)$ are the density functions for the two groups.

Then we can solve for the appropriate value of $\lambda_{C}$ that yields the desired expected proportion of total failures. We will illustrate the calculations in Example. If the censoring times are expected to increase or decrease monotonely, a Weibull censoring distribution can be used. If the censoring times occur at approximately equal intervals throughout the study, then a uniform distribution may be appropriate.

The power of the study can be calculated using the following procedure:

1. Specify the sample size for each group, $n$ and $r n$, and type I error rate (by default 0.05).

2. Specify the value for the regression parameter.

3. Assume the survival time follows the additive hazards model as in (2.1) with parameters $\beta, \theta$ and $\nu$ (prior data may be used to obtain the estimates). Choose a censoring distribution such as the exponential, Weibull or uniform.

4. Specify the overall expected proportion of failures and calculate the corresponding parameter for the chosen censoring distribution accordingly.

5. Calculate the information and variance-covariance matrix based on the specified parameters (see Appendix).

6. Obtain the noncentrality parameter $\omega$.

7. Use (2.2) to calculate power.

8. Repeat steps 5-7 by changing the sample size until the desired power is achieved.

\section{Example}

Suppose we want to design a clinical trial that the outcome will be time to relapse of leukemia with prior data in Table 1. Assume that the two groups have equal size and length of leukemia remission will be recorded and right-censored failure time data will result. Assuming the survival time follows the additive hazards model as in (2.1). Fitting the prior data, we obtained prior information about the parameter estimates: $\hat{\theta}=9.08, \hat{\nu}=1.21$ and $\hat{\beta}=-0.11$. Suppose that 
the investigators expect that overall $35 \%$ of the leukemia patients will relapse by the end of the study and the censoring times will be exponentially distributed with parameter $\lambda_{C}$. Solving

$$
0.35=\int_{0}^{\infty}\left[\frac{1}{1+r} f_{T}(t \mid z=0)+\frac{r}{1+r} f_{T}(t \mid z=1)\right] S_{C}(t) d t
$$

will yield a value of 0.0175 for $\lambda_{C}$. Table 2 lists the required sample sizes to achieve $80 \%$ power in testing $H_{1}: \beta \neq 0$.

Table 2: Total sample size to achieve $80 \%$ power

\begin{tabular}{cc}
\hline$\beta$ & total sample size \\
\hline-0.10 & 222 \\
-0.07 & 384 \\
-0.05 & 648 \\
\hline
\end{tabular}

\section{Simulation Studies}

In this section, we report the power calculations using the proposed method from two simulation studies. Each simulated dataset consisted of two groups (exposed or unexposed) according to a key covariate (coded as 0 for unexposed or 1 for exposed). The failure times are assumed to follow the additive hazards model specified in (1.2) with density function

$$
f(t ; Z)=e^{-\left(\frac{t}{\theta}\right)^{\nu}-\beta^{\prime} Z t}\left(\frac{\nu}{\theta^{\nu}} t^{\nu-1}+\beta^{\prime} Z\right) .
$$

The regression parameter and the scale and shape parameters of the baseline hazard function are denoted by $\beta, \theta$ and $\nu$, respectively. For both studies, we chose $\nu_{0}=2,1$ and 0.5 to represent increasing, constant and decreasing hazard, respectively.

For the first study, the censoring variable was assumed to follow a uniform distribution $U(0,7)$. The covariate was assumed to have a Bernoulli distribution with probability of success 0.5 . For each scenario 500 datasets were generated. Datasets were generated with $\theta_{0}=5$ and $\nu_{0}=2,1$, and 0.5 and the regression parameter $\beta_{0}=0.05,0.10$ and 0.15 . Three total sample sizes for both groups $(N)$ were chosen to approximately achieve power $0.70,0.80$ and 0.90 . The datasets were analyzed with the additive hazards model. Power was calculated for each scenario using the proposed method. To explore the extent of power loss with misspecified model, we compared the power under the additive hazards model and the routinely used semiparametric Cox proportional hazards model. Empirical power was calculated as the number of datasets resulting in the rejection of $\beta=0$ 
at a significance level $\alpha=0.05$ divided by 500 . The simulation results from the first study are presented in Table 3.

Table 3: Power calculations when censoring time is uniformly distributed

\begin{tabular}{|c|c|c|c|c|c|c|c|c|c|}
\hline \multirow{2}{*}{$\begin{array}{c}\beta_{0} \\
0.05\end{array}$} & \multicolumn{3}{|c|}{$\nu_{0}=2$} & \multicolumn{3}{|c|}{$\nu_{0}=1$} & \multicolumn{3}{|c|}{$\nu_{0}=0.5$} \\
\hline & $386^{*}$ & 516 & 730 & 834 & 1120 & 1600 & 870 & 1190 & 1710 \\
\hline & $0.708^{\S}$ & 0.810 & 0.905 & 0.697 & 0.815 & 0.895 & 0.698 & 0.793 & 0.910 \\
\hline & $0.704^{\star}$ & 0.800 & 0.902 & 0.702 & 0.800 & 0.900 & 0.702 & 0.802 & 0.902 \\
\hline & $0.482^{\dagger}$ & 0.565 & 0.684 & 0.594 & 0.692 & 0.818 & 0.467 & 0.559 & 0.687 \\
\hline & & $43.1 \% \ddagger$ & & & $49.4 \%$ & & & $55.7 \%$ & \\
\hline \multirow[t]{5}{*}{0.10} & 146 & 190 & 270 & 264 & 366 & 520 & 296 & 410 & 580 \\
\hline & 0.698 & 0.811 & 0.909 & 0.709 & 0.799 & 0.912 & 0.699 & 0.801 & 0.895 \\
\hline & 0.707 & 0.805 & 0.904 & 0.701 & 0.802 & 0.902 & 0.705 & 0.807 & 0.903 \\
\hline & 0.539 & 0.628 & 0.761 & 0.605 & 0.712 & 0.832 & 0.495 & 0.593 & 0.718 \\
\hline & & $46.4 \%$ & & & $52.2 \%$ & & & $58.3 \%$ & \\
\hline \multirow[t]{5}{*}{0.15} & 84 & 110 & 156 & 146 & 196 & 276 & 172 & 226 & 330 \\
\hline & 0.712 & 0.815 & 0.910 & 0.695 & 0.797 & 0.895 & 0.710 & 0.811 & 0.912 \\
\hline & 0.703 & 0.804 & 0.900 & 0.705 & 0.802 & 0.905 & 0.704 & 0.801 & 0.905 \\
\hline & 0.575 & 0.673 & 0.781 & 0.621 & 0.723 & 0.842 & 0.521 & 0.621 & 0.750 \\
\hline & & $48.8 \%$ & & & $54.6 \%$ & & & $60.3 \%$ & \\
\hline
\end{tabular}

* the total sample size for the two groups

$\S$ the calculated power using the proposed method

$\star$ the empirical power under the additive hazards model

$\dagger$ power under the semiparametric Cox proportional hazards model

$\ddagger$ average percent failures in the datasets.

The calculated power using the proposed method is to test $H_{0}: \beta=0$ versus $H_{1}: \beta \neq 0$ at the type I error rate $\alpha=0.05$ (two-sided). The survival function is $S(t ; Z)=e^{-\left(\frac{t}{\theta}\right)^{\nu}-\beta Z t}$ where $\nu$ denotes the shape parameter and $\theta_{0}=5$. The censoring time follows a Uniform distribution $U(0,7)$. The empirical power presented is based on 500 simulated datasets.

The second study was conducted with an exponentially distributed censoring time. This scenario corresponds to a situation where censoring times occur with a constant rate. The underlying survival model is the same as in the first study. The simulation results for the second study are shown in Table 4.

For both the simulation studies, the calculated power was reasonably close to the empirical power for all the scenarios. Misspecified Cox model resulted in substantially decreased power in all the scenarios. However, the power loss was less with constant hazards $\left(\nu_{0}=1\right)$ compared to those with either increasing or decreasing hazards. 
Table 4: Power calculations when censoring time is exponentially distributed

\begin{tabular}{|c|c|c|c|c|c|c|c|c|c|}
\hline \multirow{2}{*}{$\begin{array}{c}\beta_{0} \\
0.05\end{array}$} & \multicolumn{3}{|c|}{$\nu_{0}=2$} & \multicolumn{3}{|c|}{$\nu_{0}=1$} & \multicolumn{3}{|c|}{$\nu_{0}=0.5$} \\
\hline & $410^{*}$ & 550 & 795 & 910 & 1180 & 1760 & 900 & 1200 & 1740 \\
\hline & $0.711^{\S}$ & 0.809 & 0.908 & 0.712 & 0.794 & 0.893 & 0.698 & 0.797 & 0.910 \\
\hline & $0.703^{\star}$ & 0.801 & 0.900 & 0.705 & 0.801 & 0.900 & 0.702 & 0.804 & 0.901 \\
\hline & $0.464^{\dagger}$ & 0.547 & 0.674 & 0.596 & 0.693 & 0.815 & 0.472 & 0.563 & 0.670 \\
\hline & & $41.2 \% \ddagger$ & & & $47.3 \%$ & & & $54.1 \%$ & \\
\hline \multirow[t]{5}{*}{0.10} & 140 & 194 & 276 & 274 & 390 & 550 & 310 & 420 & 600 \\
\hline & 0.695 & 0.795 & 0.910 & 0.709 & 0.794 & 0.895 & 0.698 & 0.814 & 0.910 \\
\hline & 0.701 & 0.803 & 0.906 & 0.704 & 0.800 & 0.900 & 0.705 & 0.804 & 0.902 \\
\hline & 0.521 & 0.614 & 0.734 & 0.609 & 0.706 & 0.828 & 0.506 & 0.591 & 0.715 \\
\hline & & $43.7 \%$ & & & $49.6 \%$ & & & $56.3 \%$ & \\
\hline \multirow[t]{5}{*}{0.15} & 82 & 110 & 156 & 144 & 200 & 286 & 186 & 240 & 344 \\
\hline & 0.693 & 0.817 & 0.910 & 0.719 & 0.813 & 0.892 & 0.715 & 0.813 & 0.909 \\
\hline & 0.704 & 0.803 & 0.900 & 0.710 & 0.800 & 0.901 & 0.704 & 0.801 & 0.900 \\
\hline & 0.553 & 0.649 & 0.760 & 0.623 & 0.718 & 0.837 & 0.537 & 0.627 & 0.745 \\
\hline & & $45.6 \%$ & & & $51.5 \%$ & & & $58.1 \%$ & \\
\hline
\end{tabular}

* the total sample size for the two groups

$\S$ the calculated power using the proposed method

* the empirical power under the additive hazards model

$\dagger$ power under the semiparametric Cox proportional hazards model

$\ddagger$ average percent failures in the datasets.

The calculated power using the proposed method is to test $H_{0}: \beta=0$ versus $H_{1}: \beta \neq 0$ at the type I error rate $\alpha=0.05$ (two-sided). The survival function is $S(t ; Z)=e^{-\left(\frac{t}{\theta}\right)^{\nu}-\beta Z t}$ where $\nu$ denotes the shape parameter and $\theta_{0}=5$. The censoring time follows an exponential distribution $\exp (0.25)$. The empirical power presented is based on 500 simulated datasets.

\section{Concluding Remarks}

We propose power calculations based on an additive hazards model and a two-group comparison via the Wald test. Simulation results demonstrate that the proposed method for calculating power performs reasonably well. The proposed method can be easily generalized with a generalized Weibull as the baseline hazards. This allows more flexibility in specifying the form of the baseline hazards. This approach can also be easily extended to other parametric survival and censoring distributions.

Our approach assumes a Weilbull distribution for the baseline hazards, which specifies a parametric distribution of survival time. However, assessing this baseline hazards assumption is a challenge for real data. Therefore, a power calcu- 
lation based on the semiparametric additive hazards model is warranted which leaves the baseline hazards completely unspecified.

This method calculates the total required number of subjects. However, in a survival study, usually the number of actual death or events that must be observed is more important. It would be helpful to derive an expression for the required number of death or events under the semiparametric additive hazards model.

\section{Appendix}

Assume the additive hazards model with the baseline hazard following a Weibull distribution specified in model (2.1) in Section 2. Then the survival function of the failure time is

$$
S(t ; Z)=e^{-\left(\frac{t}{\theta}\right)^{\nu}-\beta^{\prime} Z t}
$$

The density function of the failure time is

$$
f(t ; Z)=e^{-\left(\frac{t}{\theta}\right)^{\nu}-\beta^{\prime} Z t}\left(\frac{\nu}{\theta^{\nu}} t^{\nu-1}+\beta^{\prime} Z\right) .
$$

The likelihood function

$$
\begin{aligned}
& L(X, \delta, Z ; \theta, \nu, \beta)=\prod_{i=1}^{N} S\left(x_{i}, \delta_{i}, z_{i} ; \theta, \nu, \beta\right)^{1-\delta_{i}} f\left(x_{i}, \delta_{i}, z_{i} ; \theta, \nu, \beta\right)^{\delta_{i}} \\
& =\prod_{i=1}^{N}\left\{\exp \left[-\left(\frac{x_{i}}{\theta}\right)^{\nu}-\beta^{\prime} z_{i} x_{i}\right]\right\}^{1-\delta_{i}}\left\{\exp \left[-\left(\frac{x_{i}}{\theta}\right)^{\nu}-\beta^{\prime} z_{i} x_{i}\right]\left(\frac{\nu}{\theta^{\nu}} x_{i}^{\nu-1}+\beta^{\prime} z_{i}\right)\right\}^{\delta_{i}} .
\end{aligned}
$$

To estimate $\beta, \nu$ and $\theta$, the elements of the $3 \times 1$ score vector are

$$
\begin{aligned}
& \frac{\partial \log L}{\partial \beta}=\sum_{i=1}^{N}\left\{-z_{i} x_{i}+\frac{\delta_{i} z_{i}}{\left(\frac{\nu}{\theta^{\nu}} x_{i}^{\nu-1}+\beta^{\prime} z_{i}\right)}\right\}, \\
& \frac{\partial \log L}{\partial \theta}=\sum_{i=1}^{N}\left\{\frac{\nu}{\theta}\left(\frac{x_{i}}{\theta}\right)^{\nu}-\delta_{i}\left[\frac{\left(\frac{\nu}{\theta}\right)^{2}}{\frac{\nu}{\theta}+\beta^{\prime} z_{i}\left(\frac{\theta}{x_{i}}\right)^{\nu-1}}\right]\right\}, \\
& \frac{\partial \log L}{\partial \nu}=\sum_{i=1}^{N}\left\{-\left(\frac{x_{i}}{\theta}\right)^{\nu} \log \frac{x_{i}}{\theta}+\delta_{i}\left[\frac{1+\nu \log \frac{x_{i}}{\theta}}{\nu+\beta^{\prime} z_{i} x_{i}\left(\frac{\theta}{x_{i}}\right)^{\nu}}\right]\right\} .
\end{aligned}
$$

The elements of the $3 \times 3$ observed Fisher information matrix are

$$
\frac{\partial^{2} \log L}{\partial \beta^{2}}=\sum_{i=1}^{N}\left\{\frac{-\delta_{i} z_{i}^{2}}{\left(\frac{\nu}{\theta^{\nu}} x_{i}^{\nu-1}+\beta^{\prime} z_{i}\right)^{2}}\right\}
$$




$$
\begin{aligned}
& \frac{\partial^{2} \log L}{\partial \theta^{2}}=\sum_{i=1}^{N}\left\{-(\nu+1) \nu x_{i}^{\nu} \theta^{-(\nu+2)}-\delta_{i}\left[\frac{\nu^{3} \theta^{-4}+\beta^{\prime} z_{i} x_{i}^{1-\nu} \theta^{\nu-4} \nu^{2}(\nu+1)}{\left(\frac{\nu}{\theta}+\beta^{\prime} z_{i}\left(\frac{\theta}{x_{i}}\right)^{\nu-1}\right)^{2}}\right]\right\}, \\
& \frac{\partial^{2} \log L}{\partial \nu^{2}}=\sum_{i=1}^{N}\left\{-\left(\log \frac{x_{i}}{\theta}\right)^{2}\left(\frac{x_{i}}{\theta}\right)^{\nu}\right. \\
& \left.+\delta_{i}\left[\frac{\log \frac{x_{i}}{\theta}\left[\nu+\beta^{\prime} z_{i} x_{i}\left(\frac{\theta}{x_{i}}\right)^{\nu}\right]-\left(1+\nu \log \frac{x_{i}}{\theta}\right)\left[1+\beta^{\prime} z_{i} x_{i}\left(\log \frac{\theta}{x_{i}}\right)\left(\frac{\theta}{x_{i}}\right)^{\nu}\right]}{\left[\nu+\beta^{\prime} z_{i} x_{i}\left(\frac{\theta}{x_{i}}\right)^{\nu}\right]^{2}}\right]\right\}, \\
& \frac{\partial^{2} \log L}{\partial \beta \partial \theta}=\sum_{i=1}^{N}\left\{\frac{\delta_{i} z_{i} \nu^{2} x_{i}^{\nu-1} \theta^{-(\nu+1)}}{\left(\frac{\nu}{\theta^{\nu}} x_{i}^{\nu-1}+\beta^{\prime} z_{i}\right)^{2}}\right\} \text {, } \\
& \frac{\partial^{2} \log L}{\partial \beta \partial \nu}=\sum_{i=1}^{N}\left\{\frac{-\delta_{i} x_{i} z_{i}}{\left[\nu\left(\frac{x_{i}}{\theta}\right)^{\nu}+\beta^{\prime} z_{i} x_{i}\right]^{2}}\left(\frac{x_{i}}{\theta}\right)^{\nu}\left(1+\nu \log \frac{x_{i}}{\theta}\right)\right\} \text {, } \\
& \frac{\partial^{2} \log L}{\partial \theta \partial \nu}=\sum_{i=1}^{N}\left\{\frac{1}{\theta}\left(\frac{x_{i}}{\theta}\right)^{\nu}+\frac{\nu}{\theta}\left(\log \frac{x_{i}}{\theta}\right)\left(\frac{x_{i}}{\theta}\right)^{\nu}\right. \\
& \left.-\delta_{i}\left[\frac{\frac{2 \nu}{\theta^{2}}\left[\frac{\nu}{\theta}+\beta^{\prime} z_{i}\left(\frac{\theta}{x_{i}}\right)^{\nu-1}\right]-\frac{\nu^{2}}{\theta^{3}}\left[1+\beta^{\prime} z_{i} x_{i}\left(\log \frac{\theta}{x_{i}}\right)\left(\frac{\theta}{x_{i}}\right)^{\nu}\right]}{\left[\frac{\nu}{\theta}+\beta^{\prime} z_{i}\left(\frac{\theta}{x_{i}}\right)^{\nu-1}\right]^{2}}\right]\right\} .
\end{aligned}
$$

\section{References}

Aalen, O. O. (1980). A model for non-parametric regression analysis of counting processes. In: W. Klonecki, A. Kozek and J. Rosinski (Eds.), Lecture Notes in Statistics-2, pp. 1-25. Springer, New York.

Anderson, P. K. and Gill, R. D. (1982). Cox's regression model for counting processes: a large sample study. Annals of Statistics 10, 1100-1120.

Aranda-Ordaz, F. J. (1981). On two families of transformations to additivity for binary response data. Biometrika 68, 357-363.

Aranda-Ordaz, F. J. (1983). An extension of the proportional hazards model for grouped data. Biometrics 39, 109-117.

Breslow, N. E. and Day, N. E. (1987). Statistical Methods in Cancer Research, Vol. 2. In: The Design and Analysis of Cohort Studies. International Agency for Research on Cancer, Lyon. 
Cox, D. R. (1972). Regression models and life tables. Journal of the Royal Statistical Society, Series B 34, 187-220.

Cox, D. R. (1975). Partial likelihood. Biometrika 62, 269-276.

Freireich, E. J. et al. (1963). The effect of 6-Mercaptopurine on the duration of steroid-induced remissions in acute leukemia. Blood 21, 699-716.

Gehan, E. A. (1965). A generalized Wilcoxon test for comparing arbitrarily singly-censored samples. Biometrika 52, 203-223.

Martinussen, T., Aalen, O. O. and Scheike, T. H. (2008). The Mizon-Richard encompassing test for the Cox and Aalen additive hazards models. Biometrics 64, 164-171.

McKeague, I. W. and Utikal, K. J. (1991). Goodness-of-fit tests for additive hazards and proportional hazards models. Scandinavian Journal of Statistics 18, 177-195.

Schoenfeld, D. A. (1983). Sample-size formula for the proportional-hazards regression model. Biometrics 39, 499-503.

Tibshirani, R. J. and Ciampi, A. (1983). A family of proportional- and additivehazards models for survival data. Biometrics 39, 141-147.

Tsiatis, A. A. (1981). A large sample study of Cox's regression model. Annals of Statistics 9, 93-108.

Wald, A. (1943). Tests of statistical hypotheses concerning several parameters when the number of observations is large. Transactions of the American Mathematical Society 54, 426-482.

Received August 11, 2011; accepted September 26, 2011.

\author{
Ling Chen \\ Division of Biostatistics \\ Washington University School of Medicine \\ Campus Box 8067, 660 S. Euclid Ave, St. Louis, MO 63110, USA \\ ling@wubios.wustl.edu \\ Chengjie Xiong \\ Division of Biostatistics \\ Washington University School of Medicine \\ Campus Box 8067, 660 S. Euclid Ave, St. Louis, MO 63110, USA \\ chengjie@wubios.wustl.edu
}


J. Philip Miller

Division of Biostatistics

Washington University School of Medicine

Campus Box 8067, 660 S. Euclid Ave, St. Louis, MO 63110, USA

jphilipmiller@wustl.edu

Feng Gao

Division of Biostatistics

Washington University School of Medicine

Campus Box 8067, 660 S. Euclid Ave, St. Louis, MO 63110, USA

feng@wubios.wustl.edu 